|| Print ISSN: 2589-7837 || Online ISSN: 2581-3935 ||

International Journal of Medical Science and Diagnosis Research (IJMSDR)

Available Online at www.ijmsdr.com

NLM (National Library of Medicine ID: 101738824)

\title{
To Study the Efficacy of Low Level Laser Therapy as Compared to Patellofemoral Mobilization along with Knee Strengthening Exercises (Conventional treatment) in Patients with Bilateral Patellofemoral Knee Osteoarthritis- A Randomised Split- Body Design.
}

\section{Hanisha Goyal}

Post-Graduate Student, Department of Physiotherapy, Guru Nanak Dev University, Amritsar - 143005, Punjab, India

\section{Conflicts of Interest: Nil}

Corresponding author: Hanisha Goyal

DOI: https://doi.org/10.32553/ijmsdr.v5i10.863

\begin{abstract}
:
Purpose: The main purpose of the study was to find out the effects of Low Level Laser Therapy in patients presenting with diagnosis of bilateral patellofemoral knee osteoarthritis. The objective of our research is: To improve functional outcome.

Methodology: A split-body-experimental design was done by random sampling for 4 weeks. A minimum of 20 subjects (i.e., 40 knees) were assigned for the study and low level laser therapy was applied to the right 20 knee (experimental group) and left 20 knee (control group) received conventional treatment in bilateral osteoarthritis, therefore number of knees tested were 20 for low level laser therapy and other 20 will receive conventional treatment
\end{abstract}

Result: Statistically significant improvement was indicated in respect to all parameters taken in WOMAC scale such as pain, stiffness and physical function. There was also significant decrease in swelling by both conventional and additional low level laser therapy. Improvements in all parameters of the experimental group were more statistically significant when compared to parameters of control group $(\mathrm{p}<0.05)$.

Conclusion: This study suggests that there is significant improvement with additional Low Level Laser therapy as well as with conventional treatment in patients with patellofemoral knee osteoarthritis but there is more significant improvement in experimental group in which Low level laser therapy was given along with exercise program. There is decrease in pain, swelling and stiffness. Hence, addition of Low Level laser therapy in patella-femoral knee osteoarthritis is more effective than conventional treatment.

Keywords: Patellofemoral osteoarthritis, Low Level laser therapy, knee joint, isometric strengthening.

\section{Introduction:}

Knee joint is the most complex joint of the body. It is formed by fusion of femur, tibia and patella bones. It is condylar synovial joint incorporating between the condyles of the femur and tibia, and saddle joint between the femur and patella. Patellofemoral joint is formed by the fusion of patella and femur. It consist of various ligaments such as tibial and fibular collateral ligaments, anterior and posterior cruciate ligaments, oblique popliteal ligament and arcuate popliteal ligament and primary muscles such as quadriceps femoris, popliteus, biceps femoris, semitendinosus, semimembranosus which assist in movement of knee joint. Various movements are possible at the knee joint and that are: Flexion, extension, medial and lateral rotation, locking and unlocking. As the knee joint is the weight bearing joint of the human body and helps in walking, it requires large blood supply. ${ }^{1}$ 
Osteoarthritis is a degenerative, non-inflammatory joint diseases characterized by destruction of articular cartilage of the joint and new bone formation at the joint surfaces and margins. There are various risk factors like obesity, senility, trauma, and hormonal imbalance, diseases of joints, poor posture and repetitive injuries. Osteoarthritis includes symptoms such as pain, early morning stiffness, restricted range of motion, swelling of the joints. One of the most common osteoarthritis is knee osteoarthritis as it is a weight bearing joint. It is due to the frequent need of squatting and sitting cross-legged. Patients complain of pain, limp, difficulty in descending and ascending stairs and getting up from chair. Its symptoms include pain, swelling, stiffness, clicking sound in knee osteoarthritis. ${ }^{2}$

According to Kellegren and Lawrence Radiological Grading, it is divided into 5 grades: Grade I: Doubtful narrowing of joint space and possible osteophyte lipping; Grade II: Definite osteophytes and possible narrowing of the joint space; Grade III: Moderate multiple osteophytes, definite narrowing of joint space and some sclerosis and possible deformity of the bone ends; Grade IV: Large osteophytes, marked narrowing of joint space, severe sclerosis and definite deformity of the bone ends. ${ }^{3}$

The knee joint, a complex tri-compartment structure, comprises the patello-femoral joint and the tibiofemoral joint. The coexistence of patellafemoral joint osteoarthritis and tibio-femoral joint osteoarthritis is observed in $40 \%$ of older adults with knee osteoarthritis. ${ }^{4}$ Previous studies have indicated that patello-femoral joint osteoarthritis is a major source of pain in knee osteoarthritis, and that the concomitant occurrence of patello-femoral joint osteoarthritis and tibiofemoral joint osteoarthritis causes a greater degree of pain and loss of function. ${ }^{4,5,6}$ Knee mobilizations may be beneficial for individuals with a variety of conditions, including post-operative rehab and knee osteoarthritis (OA). Patellofemoral glides are used when restriction of the patellofemoral joint causes pain or decreased overall knee ROM/function. Medial/lateral glides are utilized with the patient in supine with the knee slightly flexed. To provide a medial glide, the therapist utilizes both hands to press on the inferior and superior aspects of the medial patella and deliver a force to glide the patella in a lateral direction. Conversely, contact points on the lateral patella are used to produce a medial glide. Superior and inferior glides are used for joint play and patellar motion necessary for extension and flexion, respectively. ${ }^{7}$

Laser is also found to be effective in case of knee osteoarthritis. Low-level laser therapy (LLLT) was introduced as an alternative non-invasive treatment for osteoarthritis, but its effectiveness is still controversial. LASER stands for 'Light Amplification by Stimulated Emission of Radiation' LLLT is a non-invasive, painless and a non-thermal modality. Low Level Laser Therapy (LLLT) is a reliable, safe and effective treatment used to enhance the healing process, reduced inflammation and relieves pain in musculoskeletal and dermal conditions. It uses low levels of Laser light to modulate cellular or tissue function to achieve therapeutic benefits. ${ }^{8,9}$

\section{Materials and Methods}

Samples: Data was collected from a minimum of 20 patients (i.e. 40 knees) of age group between 45-60 years diagnosed with bilateral patellofemoral knee osteoarthritis, who were referred from other departments to Outpatient Physiotherapy Department of College of Physiotherapy, Christian Medical College and Hospital, Ludhiana. Low level laser therapy was applied to the right 20 knees and left 20 knees received conventional treatment in bilateral osteoarthritis, therefore number of knees tested was 20 for low level laser therapy and other 20 will receive conventional treatment after taking informed written consent.

Selection Criteria: Patients diagnosed with knee osteoarthritis and fulfilling the following inclusion and exclusion criteria were selected for the study after taking informed written consent. The inclusion criteria were patients (both male and female) with age group between 45-60 years diagnosed with bilateral idiopathic patellofemoral knee osteoarthritis; Grade I and II bilateral knee 
OA confirmed radiologically according to the Kellgren-Lawrence grading system. The exclusion criteria were patients with knee osteoarthritis with Kellegren-Lawrence Grade III and IV; knee joint disease other than Osteoarthritis; intra-articular corticosteroid injection in knee joint; any previous knee surgery.

Patients attended four weekly physical therapy sessions during a eight week period. Patients with bilateral knee osteoarthritis who came to department of physiotherapy and Split- body experimental research design was used to separate the treatment of knees:

For the left knees (control group): Patients treated by patellofemoral mobilization, hydro collateral packs and undergone an exercise session consisting of warm up exercises, active range of motion exercises for the knee muscles, strengthening exercises for the hip and knee muscles, muscle stretching of lower limb. ${ }^{10}$
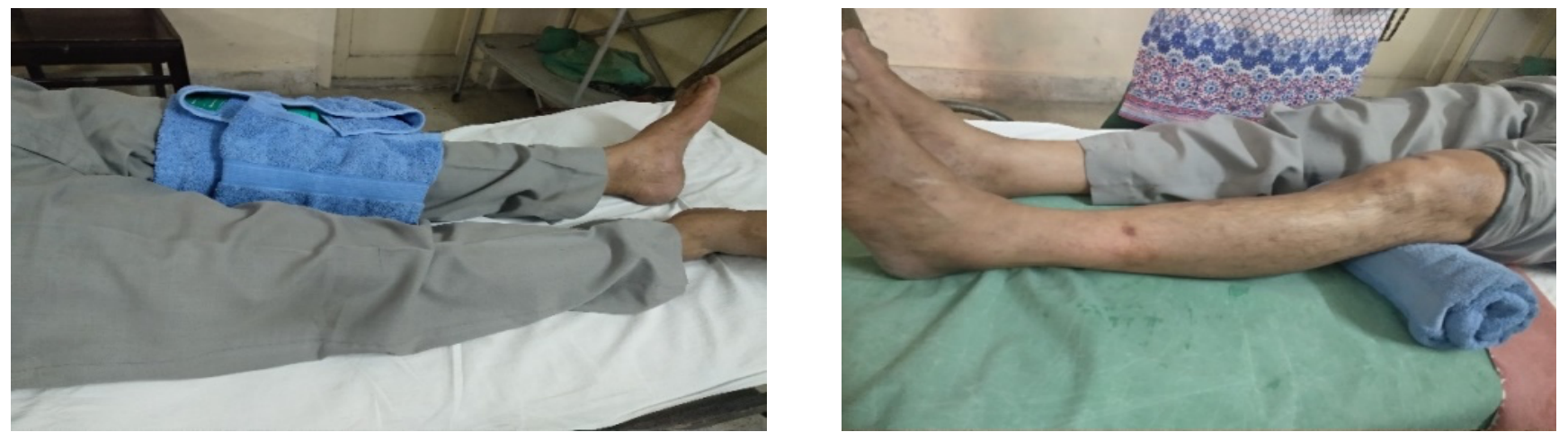

Fig. 1: Left knees treated with Hydro-collateral packs.

Fig. 2: Left knees treated with quadriceps strengthening exercises

For the right knees (experimental group): Patients were instructed to sit in long sitting position on the treatment table. The right knee was exposed and treated with low level laser therapy followed by exercise session consisting of warm up exercises, active range of motion exercises for the knee muscles, strengthening exercises for the hip and knee muscles, muscle stretching of lower limb. The study was approved by Institutional Ethical Committee.

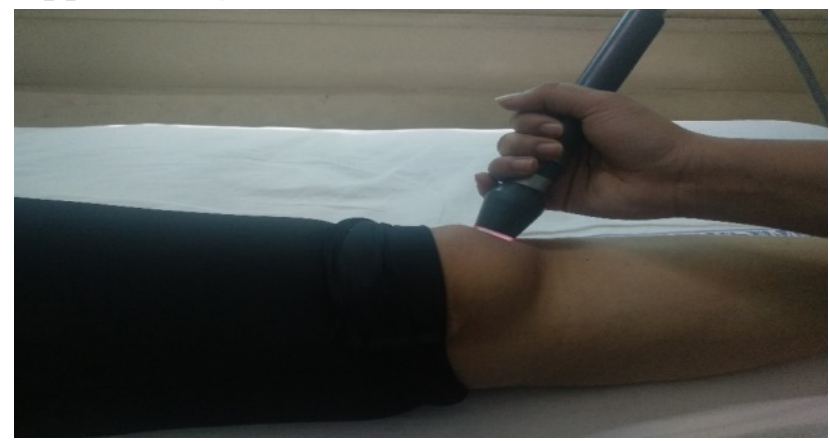

Fig. 3: Right knees treated with Low Level Laser Therapy

\section{Outcome Measure:}

The outcome measures included at the zero week, after the $2^{\text {nd }}$ week and $4^{\text {th }}$ week of treatment, all patients were evaluated by the Western Ontario McMasters Osteoarthritis Index (WOMAC score). The Western Ontario and McMaster Universities Arthritis Index (WOMAC) was

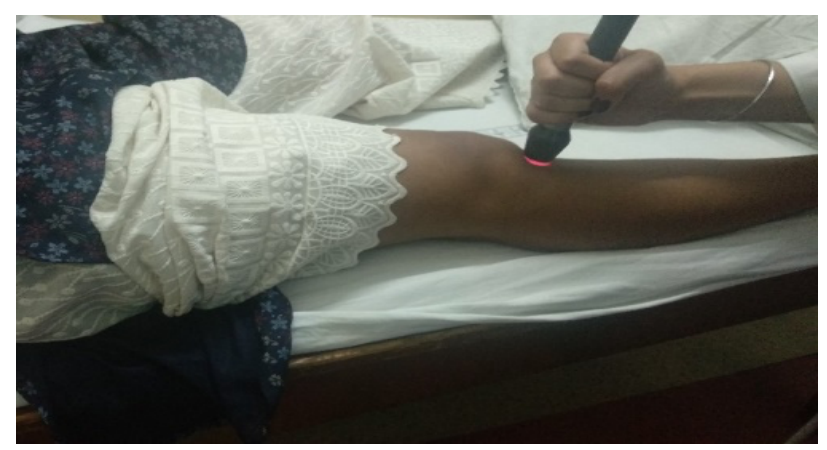

Fig. 4: Right knees treated with Low Level Laser Therapy

developed in 1982 and is widely used in the evaluation of Hip and Knee Osteoarthritis. It is a self-administered questionnaire consisting of 24 items divided into 3 subscales ${ }^{11}$

- Pain (5 items): during walking, using stairs, in bed, sitting or lying, and standing upright 
- Stiffness (2 items): after first waking and later in the day.

- Physical Function (17 items): using stairs, rising from sitting, standing, bending, walking, getting in / out of a car, shopping, putting on / taking off socks, rising from bed, lying in bed, getting in / out of bath, sitting, getting on / off toilet, heavy domestic duties, light domestic duties.

It offers following response score ranging from none to extreme. 'None' is scored 0, 'Mild' is scored 1, 'Moderate' as 2, 'Severe' as 3 and 'Extreme' as 4 and Numeric version of the visual analog scale (VAS scale).

\section{Low Level Laser Therapy}

A $3 b$ laser was used in this procedure as a source of low-power laser. Patients received laser therapy with a probe with a wavelength of $810 \mathrm{~nm}$ and 50 $\mathrm{mW}$ output power in pulse radiation mode of: Frequency: 300, Density: $0.05 \mathrm{~W} / \mathrm{cm}^{2}$, Dosage: $6 \mathrm{~J} / \mathrm{cm}^{2}$, Area: $1 \mathrm{~cm}^{2}$. In Low level laser therapy when laser beam penetrates in the skin, chromophores of skin absorbs the photons and Photobiomodulation occur.

Photobiostimulation: increases cell metabolism, improves blood circulation, increases ATP production, increases collagen and protein production, activation and proliferation of fibroblasts.

Photobioinhibition: reduces specific inflammatory markers (prostaglandins E2, interleukin), alters prostaglandin and endogenous opioid production, increases pain threshold, increases activity of $\mathrm{Na}$ and $\mathrm{K}$ pump. ${ }^{12}$

\section{Patellofemoral Mobilization:}

Medial/lateral glides were utilized with the patients in supine with the knee slightly flexed. ${ }^{7}$

\section{Active Range of Motion and Warm up exercises}

Active range of motion and warm up exercises included knee flexion, knee extension in sitting position, straight leg raises, side leg raises and prone leg raise.

\section{Strengthening exercises of Hip and Knee joint}

Strengthening exercises included quadriceps strengthening exercises, hamstring strengthening exercises, vastus medialis oblique strengthening exercises and straight leg raises.

\section{Stretching exercises of lower limb}

Stretching exercises of lower limb included quadriceps stretching exercises, Hamstring stretching exercises and Tendoachillis stretch.

\section{Statistical Analysis}

The categorical data was presented as Count (percentage) and continuous variables were presented as Mean \pm S.D or Median variables. Chi square test was used to find the association of categorical variables. Independent t-test or MannWhitney $U$ test was used to find the significance difference of NPRS (Numeric version of the visual analog scale VAS) score between two groups. The improvement in NPRS score from pre to post was analyzed using paired t-test in both the groups. The $\mathrm{P}$ value $<0.05$ will be considered significant. All statistical analysis will be performed using SPSS (Statistical Packages for Social Sciences, version 21.0. Armonk, NY: IBM corp.).

\section{Result}

A minimum of 20 subjects (i.e. 40 knees) who met the inclusion criteria were evaluated aged between 45-60 years and 20 left knees were given conventional therapy and 20 right knees were given additional low level laser therapy and all of them completed the study period.

Statistically significant improvement was indicated in respect to all parameters taken in WOMAC scale such as pain, stiffness and physical function. There was also significant decrease in swelling by both conventional and additional low level laser therapy. Improvements in all parameters of the experimental group were more statistically significant when compared to parameters of control group $(p<0.05)$. Table 1 showed the comparison of score between right and left knee at different intervals and compared to baseline and week 2 , week 4 show significant 
improvements in both right and left knees i.e. $p<$ 0.001 .

Compared to baseline, at week 2 and at week 4, significant, large improvement was observed early in experimental group than control group. The score from the baseline gradually decreased till week 4 in both experimental and control group (Table 2). The results of the Chi square test were used to find the association of categorical variables. Independent t-test was used to find the significance difference of NPRS score between two groups. The improvement in NPRS score from pre to post was analyzed using paired t-test in both the groups. The $\mathrm{P}$ value $<0.05$ will be considered significant.

\begin{tabular}{|l|l|l|l|l|}
\hline \multicolumn{5}{|l|}{ Table 1: Comparison of score between Right and left knee at different time intervals } \\
\hline & $\begin{array}{l}\text { Total } \\
(\mathbf{n}=\mathbf{4 0})\end{array}$ & $\begin{array}{l}\text { Right } \\
(\mathbf{n = 2 0})\end{array}$ & $\begin{array}{l}\text { Left } \\
(\mathbf{n = 2 0})\end{array}$ & \\
\hline & Mean \pm S.D & Mean \pm S.D & Mean \pm S.D & p-value \\
\hline Baseline & $46.20 \pm 13.27$ & $45.15 \pm 14.50$ & $47.25 \pm 12.19$ & 0.623 \\
\hline Week 2 & $38.65 \pm 12.13$ & $35.15 \pm 12.04$ & $42.15 \pm 11.46$ & 0.067 \\
\hline Week 4 & $30.25 \pm 10.95$ & $24.85 \pm 7.51$ & $35.65 \pm 11.33$ & $\mathbf{0 . 0 0 1}$ \\
\hline
\end{tabular}

Table 2: Improvement in score in both Right and left knee at different time intervals

\begin{tabular}{|l|l|l|l|l|}
\hline NPRS score & Baseline & Week 2 & Week 4 & \\
\hline & Mean \pm S.D & Mean \pm S.D & Mean \pm S.D & p-value \\
\hline Control & $45.15 \pm 14.50$ & $35.15 \pm 12.04$ & $24.85 \pm 7.51$ & $<\mathbf{0 . 0 0 1}$ \\
\hline Experiment & $47.25 \pm 12.19$ & $42.15 \pm 11.46$ & $35.65 \pm 11.33$ & $<\mathbf{0 . 0 0 1}$ \\
\hline
\end{tabular}

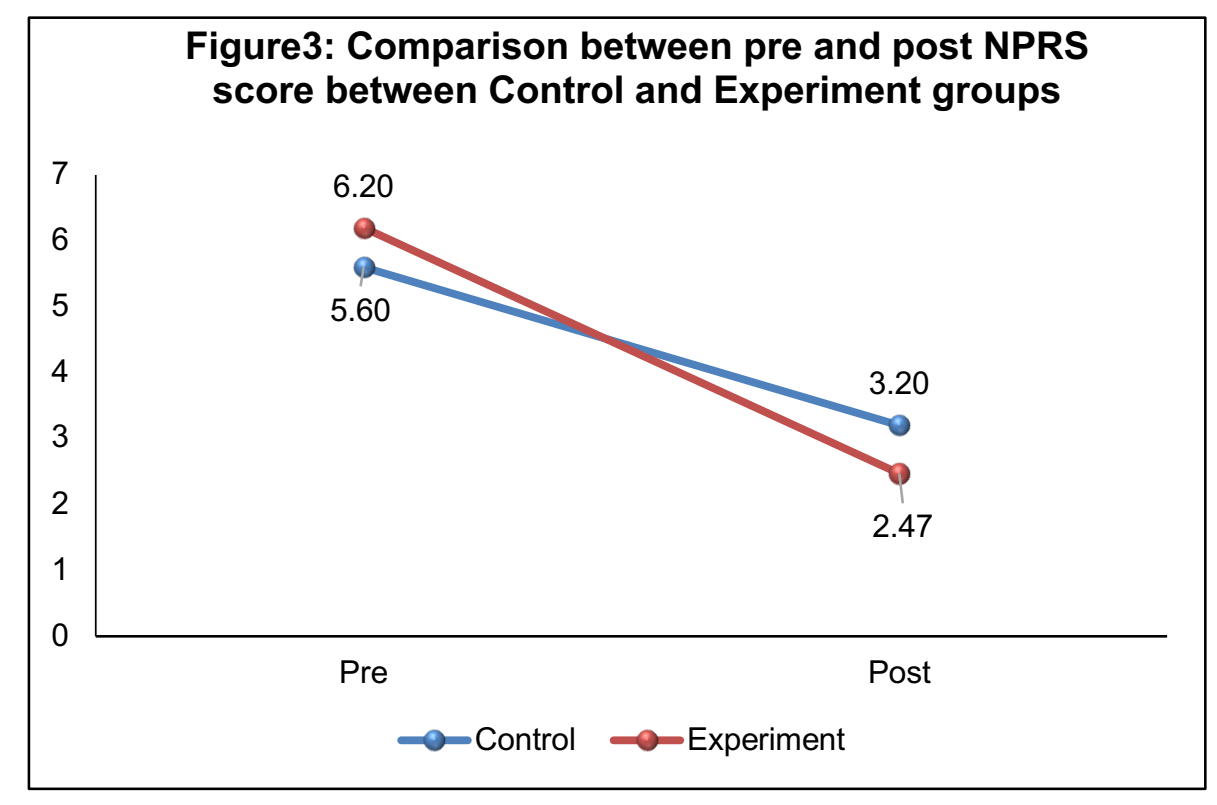

\section{Discussion}

The aim of the present study was to find out the efficacy of low level laser therapy as compared to patella-femoral mobilization along with knee strengthening exercises in patients with bilateral patellofemoral knee osteoarthritis. In this study 20 patients (40 knees) diagnosed with bilateral patellofemoral osteoarthritis were treated with conventional treatment and with additional low laser therapy treatment. By the use of split body design patients were divided in control group and 
experimental group. For The Left Knee (Control Group): Patients were treated by patellofemoral mobilization, hydro collateral packs and undergone an exercise session consisting of warm up exercises, active range of motion exercises for the knee muscles, strengthening exercises for the hip and knee muscles, muscle stretching of lower limb. For The Right Knee (Experimental Group): Patients were instructed to sit in long sitting position on the treatment table. The right knee was exposed and treated with low level laser therapy followed by exercise session consisting of warm up exercises, active range of motion exercises for the knee muscles, strengthening exercises for the hip and knee muscles, muscle stretching of lower limb.

David Ip et al found improvement in osteoarthritic knee pain with low laser therapy and concluded that it is effective in elderly patients with osteoarthritis. ${ }^{13}$ In other study D. Beulah Jebakani et al found improvement with therapeutic exercise program that includes warm up period, active range of motion exercises for the knee, muscles strengthening exercises for the hip and knee, muscle stretching for the lower limbs, balance exercises and cool down period reduced pain and improved physical disability in adults with knee osteoarthritis. ${ }^{14}$ Parisa Nejati et al also showed the effectiveness of exercise therapy on knee osteoarthritis and found that adding exercise to other noninvasive methods that routinely used for knee osteoarthritis have boosting effect in relieving pain and improving knee function. ${ }^{15}$ Patricia Pereira Alfredo et al showed effectiveness of low level laser therapy associated with exercises in knee osteoarthritis. Their findings suggest that low level laser therapy when associated with exercises is effective in yielding pain relief, function and activity in patients with knee osteoarthritis. ${ }^{16}$

In present study there is significant improvement was observed in control group and experimental group in respect to all parameters taken in WOMAC scale such as pain, stiffness and physical function. There was also significant decrease in swelling by both conventional and additional low level laser therapy. Improvements in all parameters of the experimental group were more statistically significant when compared to parameters of control group.

\section{Conclusion}

This study suggests that there is significant improvement with additional Low Level Laser therapy as well as with conventional treatment in patients with patellofemoral knee osteoarthritis but there is more significant improvement in experimental group in which low level laser therapy was given along with exercise program. There is decrease in pain, swelling and stiffness. Hence, Addition of low level laser therapy in patellofemoral knee osteoarthritis is more effective than conventional treatment.

\section{Acknowledgements}

The author is thankful to all the subjects for their cooperation in the study.

\section{Conflicts of interest}

There are no conflicts of interest.

\section{References}

1. Chaurasia B, Garg K, Mittal P, Chandrupatla M. BD Chaurasia's Human anatomy regional and applied dissection and clinical: lower limb abdomen and pelvis. 6th ed. New Delhi: CBS Publishers \& Distributors Pvt Ltd; 2004. p. 140-146.

2. Ebnezar J, John R. Textbook of orthopedics. 5th ed. Jaypee Publishers.; 2016. p. 658-666.

3. Joshi J, Kotwal P. Essentials of orthopaedics and applied physiotherapy. 3rd ed. New Delhi: Elsevier Publishers; 2016. p. 371-374.

4. Farrokhi S, Piva SR, Gil AB, Oddis CV, Brooks MM, Fitzgerald GK. Association of severity of coexisting patellofemoral disease with increased impairments and functional limitations in patients with knee osteoarthritis. Arthritis Care Res (Hoboken). 2013; 65(4):544-551.

5. Hinman RS, Crossley KM. Patellofemoral joint osteoarthritis: an important subgroup of knee osteoarthritis. Rheumatology (Oxford). 2007; 46(7):1057-1062. 
6. Lankhorst NE, Damen J, Oei EH, Verhaar JAN, Kloppenburg M, Bierma-Zeinstra SMA, van Middelkoop M. Incidence, prevalence, natural course and prognosis of patellofemoral osteoarthritis: the Cohort Hip and Cohort Knee study. Osteoarthritis Cartilage. 2017 May; 25(5):647-653.

7. Classens G. Michigan state university- Patellar Mobilizations [Internet]. Youtube.com. 2011 [cited 2011 Dec 1]. Available from: http://www.youtube.com/watch? $\mathrm{v}=\mathrm{FcU}$ $7 \mathrm{Kc} 1 \mathrm{FtnI}$

8. Chippala P. Essentials of Electrotherapy. 1st ed. AITBS Publishers; 2017.

9. Tascioglu F, Armagan O, Tabak Y, Corapci I, Oner C. Low power laser treatment in patients with knee osteoarthritis. Swiss Medical Weekly. 2004 May 1; 134(17-18):254-258.

10. Gardiner M. The principles of exercise therapy. 4th ed. Delhi: CBS Pub. and Distributors; 1985.

11. WOMAC Osteoarthritis Index [Internet]. Physiopedia. 2013 [cited 2013 Jul 12]. Available from: http://www.rheumatology.org /practice/clinical/clinicianresearchers/outcome s-instrumentation/WOMAC.asp

12. Soleimanpour H, Gahramani K, Taheri R, Golzari SE, Safari S, Esfanjani RM, Iranpour
A. The effect of low-level laser therapy on knee osteoarthritis: prospective, descriptive study. Lasers in Medical Science. 2014 Sep; 29(5):1695-1700.

13. Ip D. Does addition of low-level laser therapy (LLLT) in conservative care of knee arthritis successfully postpone the need for joint replacement? Lasers in Medical Science. 2015; 30(9):2335-2339.

14. Jebakani D, Sethu G, Pahinian A, Devi R, Kotian S, Sams L. Effects of therapeutic exercises on pain and physical disability in adults with knee osteoarthritis. Asian Journal of Scientific Research. 2014; 8(1):74-79.

15. Nejati P, Farzinmehr A, Moradi-Lakeh M. The effect of exercise therapy on knee osteoarthritis: a randomized clinical trial. Medical Journal of the Islamic Republic of Iran. 2015 Feb 25; 29:186.

16. Alfredo P, Bjordal J, Dreyer S, Meneses S, Zaguetti G, Ovanessian V, Fukuda T, Junior $\mathrm{W}$, Martins $\mathrm{R}$, Casarotto $\mathrm{R}$, Marques A. Efficacy of low level laser therapy associated with exercises in knee osteoarthritis: a randomized double-blind study. Clinical Rehabilitation. 2011; 26(6):523-533. 